\title{
Roger Keil \\ David Harvey und das Projekt einer materialistischen Stadttheorie
}

David Harvey gehört seit fast zwei Jahrzehnten zu den einflußreichsten Theoretikern der »Neuen Stadtforschung «". Bekannt geworden als Autor eines Lehrbuchs der positivistischen Geographie (Harvey, 1969), wurde er zum enfant terrible dieser traditionalistischen Disziplin, als er in Social Justice and the City (1973 erstmals einen marxistischen Ansatz wählte (cf .Badcock, 1984). Dieses Buch ist dann auch in der Folge wegen seiner explosiven Wirkung innerhalb der Geographie oft als sein wichtigstes angesehen worden. Verglichen mit späteren Schriften Harveys war Social Justice and the City eine zögerliche Annäherung an eine materialistische Position in der Geographie. Seine Entwicklung von einer liberal-humanistischen zu einer marxistischen Perspektive, die er noch als mögliche Varianten einer Lösung der sozialen Frage im räumlichen System der Stadt nebeneinander stehen läßt, organisiert die Struktur des Buches. Harveys Positionen ähnelten denen des französischen Marxisten Lefebvre, hoben sich jedoch von der strukturalistisch-althusserianischen Vorgehensweise Manuel Castells' ab, der in den 70er Jahren die Standards in der marxistischen Stadttheorie setzte (cf. Castells, 1977)' Über das folgende Jahrzehnt jedoch, bis zur Veröffentlichung von The Limits to Capital (1982), etablierte sich Harvey als Vordenker einer »neo-orthodoxen« (Katz, 1986) politischen Ökonomie der Stadt und des Raums. In einer Reihe von Artikeln entwickelte er eine auf einer kritischen Interpretation der Marxschen Theorie der Grundrente basierende Position mit dem Ziel, den historischen Materialismus mit einer räumlichen Dimension auszustatten.

The Limits to Capital, von ihm selbst als festgehaltene »Leseerfahrung《 seines Marxstudiums apostrophiert, trägt die verschiedenen Versuche Harveys zusammen, mit einigen »empty boxes « in der Urbanisierungstheorie zu Rande zu kommen. Das Resultat ist eine stringente materialistische politische Ökonomie des Raums, in der die grundlegenden Kategorien der Marxschen Theorie expliziert mit Harveys Interpretation der Bedeutung räumlicher Konfigurationen für die Gesellschaftstheorie verknüpft werden. ${ }^{2}$

Im Vorfeld von The Limits to Capital hatte Harvey eigne Kategorien entwickelt, mit denen er sich einer umfassenden Theorie der Urbanisierung näherte. Studien zur Rolle des Finanzkapitals (1974 mit Chatterjee), zur Geograpie der kapitalistischen Akkumulation (1975a), zur Urbanisierung in den USA (1975b), zur Rolle des Klassenkampfs in der baulichen Umwelt (1977), zum »städtischen Proze $\beta \ll^{3}$ unter kapitalistischen Bedingungen (1978) und zur räumlichen Krisenregulierung (»spatial fix «, 1981) legten Harveys wichtigste Konzepte dar. Im Laufe dieser Entwicklung wandte sich Harvey von der Verwendung marxistischer Konzepte für Geogra-

* Ich danke Joachim Hirsch, Margit Mayer und Walter Prigge für ihre kritischen Kommentare zu einer früheren Version dieses Papiers. 
phie und Geschichte des Urbanisierungsprozesses zu der Vorstellung, daß der Verstädterungsprozeß selbst als zentrale Komponente der Kapitalakkumulation fungiert. Darin folgt er Lefebvre, der das Überleben des Kapitalismus im 20. Jahrhundert auf dessen Fähigkeit zurückführt, den Raum als aktives Medium seiner Erhaltung und Konsolidierung zu nutzen (Lefebvre, 1976). Hier besteht eine Beziehung zu Imperialismus- und Neokolonialismustheorien, die ebenfalls unter der Prämisse operieren, daß »das Überleben des Kapitalismus... nur durch die Transformation von Raumbeziehungen und die Entstehung spezifischer geographischer Strukturen « (z.B. Zentrum und Peripherie) gewährleistet werde (1985c: 142).

1985 erschienen zwei Bände Harveys zur Geschichte der kapitalistischen Urbanisierung: The Urbanization of Capital (1985a) und Consciousness and the Urban Experience (1985b). In diesen Sammlungen von - zum Teil bereits anderswo publizierten — Essays beschäftigt sich Harvey mit dem am Ende von The Limits to Capital gestellten Problem, wie die Theorie der Urbanisierung und die (erlebte) historische Geographie dieses Prozesses konzeptionell zu verknüpfen seien. Im ersten Band (1985a) präzisiert er seine Vorstellungen zum städtischen Prozeß als materiellem Vorgang, während er sich im zweiten Band (1985b) mit der Produktion und Urbanisierung des Bewußtseins auseinandersetzt. Insbesondere Themen aus dem zweiten Band nimmt er schließlich in seinem jüngsten, in diesem Heft abgedruckten Aufsatz zur flexiblen Akkumulation durch Urbanisierung und zur Funktion der Postmodeme nochmals auf.

Auf den folgenden Seiten soll nun versucht werden, einige der Kerngedanken Harveys vor allem aus seinen neueren Veröffentlichungen, nachzuzeichnen. Es ist dabei beabsichtigt, die Lücke, die durch die bisher dürftige Veröffentlichungssituation von Harveys Arbeiten in deutscher Sprache (vgl. dazu die Sammelbände von Mayer/Roth/Brandes 1978 und von Kraemer/Neef 1985) entstanden ist, ein wenig zu schließen. Schon das Volumen des Harveyschen Werkes verbietet aber den Anspruch auf Vollständigkeit der Betrachtung im hier gestellten Rahmen. Trotz meiner Bemühungen um eine repräsentative und an aktuellen Theorieproblemen gemessene Auswahl von Themen, wird sich daher ein selektiver Zugriff nicht vermeiden lassen.

\section{Raum/Zeit/Akkumulation}

Für die meisten Vertreter der neuen Stadttheorie in den 70er Jahren war es vordringlich, sich von den hegemonialen humanökologischen Positionen der Chicago School of Sociology und ihren Epigonen zu lösen, deren »Fetischismus des Raums « den Blick auf die sozialen Verhältnisse zu versperren drohte. Autoren wie Castells $(1977)^{4}$ betonten die Abhängigkeit räumlicher Strukturen von sozialen, ökonomischen, politischen und ideologischen Prozessen, »Das Städtische « ist aus dieser Perspektive ideologisches Konstrukt, das lediglich als Ensemble von Prozessen der »kollektiven Konsumtion « Forschungsgegenstand sein kann. Saunders (1981) wandte sich gänzlich gegen die Vorstellung eine Integration von Raum und Gesellschaft als realem Objekt gesellschaftswissenschaftlicher Analyse und plädierte für eine nicht-räumliche Stadtsoziologie. In den letzten Jahren gewannen jedoch solche Positionen in der Stadttheorie an Boden, die die Frage des Verhältnisses von Raum und Gesellschaft differenzierter angin- 
gen. ${ }^{5}$ Es begann sich die Einsicht durchzusetzen, daß die »historische Periodizität der kapitalistischen Entwicklung mit der Abfolge von Räumlichkeiten und geographischen Landschaften einhergeht« (Soja, 1984: 28).

Für den Geographen Harvey stand das Problem der theoretischen Vermittlung des historischen Verhältnisses von Raum und Zeit oder Räumlichkeit und Periodizität also der historischen Geographie, von Haus aus im Mittelpunkt. Sein Interesse galt dabei der Bedeutung des Raums für den kapitalistischen AkkumulationsprozeB: Die Aufeinanderfolge der sozialen Produktionsformen in der Geschichte - auf Basis der ökonomischen und politischen Revolutionierung der Produktivkräfte und Produktionsverhältnisse - schuf mit einer neuen Periode auch jeweils eine neue Räumlichkeit. Der Marxsche Satz, daß die Menschen ihre Geschichte zwar selbst machen, nicht jedoch aus freien Stücken, erhält aus dieser Sicht eine neue Dimension:

$\gg$ Our historical geography is always ours to make. But the conditions under which we seek to make that historical geography are always highly structured and constrained« (Harvey, 1985a: 223).

Der Akkumulationsprozeß des Kapitals ist immer untrennbar ein raum/zeitlicher: Für das Kapital entstehen daraus gewisse Schranken, beispielsweise in der Anwendung der Ware Arbeitskraft. Die zeitlichen Hindernisse zur totalen Ausbeutung dieser besonderen Ware (cf. Storper/ Walker, 1983) sind hinlänglich bekannt. Sie bestehen sowohl in der absoluten Länge des Lebens, in der Produktionszeit (Erziehung) und der täglichen Reproduktionsnotwendigkeit ihres Trägers, des Menschen. Selten werden jedoch die räumlichen Limitationen genannt. Diese bestehen in der Besonderheit eines jeden lokalen Arbeitsmarkts, der auch im Zeitalter der Globalisierung kapitalistischer Produktion erhalten bleiben muß: Auf der weltweiten Suche nach oder bei der Schaffung von spezifischen Produktionsbedingungen vertraut das Kapital auf räumlich segmentierte und qualitativ differente Arbeitsmärkte. So willkommen diese Konfigurationen den Akkumulationsinteressen des Kapitals zu bestimmten Zeitpunkten jedoch sein mögen, so sehr stehen sie ihnen in einer anderen Periode im Wege und müssen zerstört oder restrukturiert werden (cf. Harvey, 1985a: 127 ff; Scott/Storper, 1986). Im raum/zeitlichen Prozeß der Akkumulation schafft sich das Kapital sein Gegenbild in einer physischen Landschaft:

$\gg$ The very production of this landscape, so vital to accumulation, is in the end antithetical to the tearing down of spatial bariers and the annihilation of space by time« (Harvey, 1985a: 43).

Dieser Widerspruch führt zu einem ständigen Kampf um die zweckmäßigste räumliche Ausformung des Akkumulationsprozesses, zum Kampf zwischen einem »übelieferten « und einem »geplanten « Raum (Lipietz, zitiert in Soja, 1985: 113). Für begrenzte Zeiträume stellt sich eine »strukturierte Kohärenz« her, in der sich der Zusammenhalt der sozialen Form manifestiert: die Verbindung der herrschenden Form der Technologie sowohl der Produktion als auch der Konsumtion mit den herrschenden Klassenverhältnissen in einem stadtregionalen Kontext (Harvey, 1985a: 126). Der temporären Stabiltät der »strukturierten Kohärenz« wirkt in jedem Zeit-Raum die Dynamik der historischen Geographie des Kapitalismus entgegen. Sie präsentiert sich als veränderbares und selbst in den Prozeß eingreifendes »Objekt theoretischer Untersuchung« und als »Nexus politischer Aktion« (Harvey, 1985a: 64).

Für Harvey ist der Raum eine Schlüsselkategorie im Prozeß der kapitalistischen Akkumulation. Er nimmt Levebvres Frage auf, welche Systematik sich hinter dem Bedeutungswachstum 
von Spekulation, Bawwirtschaft und Immobiliensektor in der »neokapitalistischen « Gesellschaft verbirgt (Lefebvre, 1972). Lefebvre hatte vermutet, daß es sich bei den mit der Grundrente (also mit der Distribution des Raums) verbundenen Sektoren der Ökonomie um einen Regelkreis dreht, der parallel zur Industriepolitik verläuft« und dessen Rolle wichtiger wird (Lefebvre, 1972: 169 f.): In Lefebvres Vorstellung wird die Phase der Industriellen Revolution im 20. Jahrhundert von der Phase der Urbanen Revolution überwunden:

$\gg I m$ gleichen Maße wie der Hauptregelkreis - laufende industrielle Produktion 'beweglicher' Güter - an Schwung verliert, nehmen die Investitionen auf dem sekundären, dem Immobiliensektor zu « (Lefebvre, 1972: 170).

Die Entwicklung von der industriellen zur urbanen Phase etabliert auch den städtischen Raum, das urbane Ensemble, als das Herzstück der gesellschaftlichen Dynamik. Die Stadt, die nichts und doch alles schafft, die bauliche Umwelt, rückt in den Mittelpunkt der Geschichte (Lefebvre, 1972: 127): Während Lefebvre dies vornehmlich als säkulare Tendenz versteht, betont Harvey zunächst, daß der Charakter der Investitionen in den sekundären Kapitalkreislauf, i.e. in die bauliche Umwelt, primär krisenbedingt, also zyklisch ist. Jede der globalen Krisen des Kapitalismus sei von massiven Kapitalbewegungen in die bauliche Umwelt begleitet gewesen, in einem verzweifelten letzten Versuch, der Überakkumulation Herr zu werden. Als Beispiel nennt er den Boom in der Immobilienwirtschaft vieler entwickelter kapitalistischer Länder von 1969-1973 (Harvey, 1985a: 20) ${ }^{6}$.

Harveys Überlegungen gehen von dem widersprüchlichen Charakter des »spatial fix«aus. Die Limitationen der kapitalistischen Akkumulation in Raum und Zeit können demnach vorübergehend aufgefangen werden, indem »Raum durch die Produktion von Raum überwunden wird «, wenn überschüssiges Kapital in die bauliche Umwelt investiert wird. Dadurch werden jedoch die Widersprüche des Akkumulationsprozesses nur verlagert, nicht gelöst. Sie werden vielmehr über eine größere Breite verteilt (Harvey, 1985a: 60). Die räumliche »Lösung« führt also zu neuen, größeren Widersprüchen im nächsten Zyklus, in der nächsten Krise. Damit erstellt Harvey ähnlich Lefebvre ein säkulares Entwicklungsmodell der kapitalistischen Produk tionsweise, das an die Produktion und Zerstörung räumlicher Verhältnisse gekoppelt ist. In dem Maße wie der städtische Raum - ganz wie der darin stattfindende Massenkonsum, der auch den Raum massenhaft konsumiert - zum wichtigsten Faktor des Überlebens des Kapitalismus wird, wird er zum »Lokus für die kontrollierte Reproduktion der gesellschaftlichen Verhältnisse« (Harvey, 1985a: 88). Und weiter:

"Durch die Urbanisierung des Kapitals selbst werden Surplusse mobilisiert, produziert, absorbiert und angeeignet, und durch städtischen Zerfall und Degradierung werden sie entwertet und zerstört« (1985a: 222).

\section{Spatial Fix und Postmoderne}

Die zunehmende Bedeutung des sekundären Kapitalkreislaufs hat Verschiebungen im Gefüge der Klassenstruktur und des Staates zur Folge. Fortwährende Vergesellschaftung der Produktion, Bedeutungszuwachs des Finanzkapitals? Imperialismus als Lebenslogik des Kapitals, sind Aspekte der Aufhebung der kapitalistischen Produktionsweise selbst (Harvey, 1985a: 85). 
Neue Produktions- und Konsumtionsmodi werden von der expansiven Dynamik des Kapitalismus hervorgebracht. Damit wandelt sich der Prozeß der Verstädterung in fundamentaler Weise: Einst Ausdruck für die Produktionsnotwendigkeiten der kapitalistischen Klasse, wird er zunehmend zum Symbol der Macht des Finanzkapitals und des Staates über den gesamten gesellschaftlichen Produktionsprozeß (Harvey, 1985a: 88). Die Bedeutung des Staates und des Finanzkapitals nimmt zu, weil die anarchische Konkurrenz zwischen den produktiven Kapitalen den Kapitalismus immer häufiger lahmlegt und die Schaffung bestimmter struktureller Voraussetzungen zur Akkumulation (z.B. bauliche Umwelt) behindert. Koordination des produktiven und des Bankkapitals im Finanzkapital und dessen politische Organisation im Staat werden zu den entscheidenden Voraussetzungen der Fortdauer des Akkumulationsprozesses. Sowohl auf der Ebene der Klassenformation und der Bündnisse zwischen Klassen als auch auf der politischen Ebene der Konstitution des Staates haben wir es dabei mit zunehmend krisenhaften und widersprüchlichen Strukturen zu tun, deren Instabilität das kapitalimmanente Feld politischer Auseinandersetzungen definiert (cf. Harvey, 1982, Kapitel 10).

Die hier skizzierte Dynamik der Kapitalakkumulation schuf einen neuen Urbanisierungstyp: Indem der Konsumismus zum tragenden Pfeiler der keynesianischen Ökonomie wurde, entwickelte sich die Stadt vom »workshop for production « zum »consumption artifact« (Harvey, 1975: 139) ${ }^{8}$. In der Stadt als Konsumtionszentrum sind beide Besonderheiten des keynesianischen Kapitalismus präsent: die Atomisierung der gesellschaftlichen Reproduktion (Einfamilienhaus, Automobil etc.) und die Organisation und Bereitstellung der baulichen Umwelt für die Konsumtion, die die wachsende Durchstaatlichung der Gesellschaft begründet. Die private Konsumtion ist das Kernstück der keynesianisch/fordistischen Formation, während die Schaffung der dafür notwendigen baulichen Umwelt durch den Kapitalfluß in den sekundären Kreislauf - unter der Ägide des Finanzkapitals und des Staates - gewährleistet wird. In der Landschaft der fordistischen Stadt manifestiert sich der gesellschaftliche Vormarsch der Finanzform des Kapitals in den fortgeschrittenen kapitalistischen Ländern (Harvey, 1985a: 88). Besonderheit dieser Entwicklung ist, daß die Durchsetzung der Finanzform des Kapitals nicht lediglich eine andere bauliche Umwelt schafft als die herkömmliche industrielle Form, sondern vielmehr, daß die Produktion des Raumes selbst zur Substanz der Akkumulation des Kapitals wird. Die Schaffung baulicher Umwelt für die Konsumtion wird so zur Existenzvoraussetzung des Kapitalismus -- wie das für die Parallelisierung von gesellschaftlicher Produktion und privater Konsumtion auch behauptet wird (cf. Aglietta, 1979; Hirsch/Roth, 1986). Die Tendenz zur »Wertrealisierung ohne Produktion «, die letztlich eine Sterilisierung des kapitalistischen Systems zur Folge hat, findet in der gesellschaftlichen Produktion des Raum für den Zweck der Konsumtion und in der Explosion des privaten Konsums ihre Entsprechung. Erscheint die keynesianische Stadt dabei als Inkarnation des »postindustriellen« Kapitalismus (Harvey, 1985a: 211), so ist sie doch nur denkbar als Resultat der Massenproduktion, des Hyperindustrialismus. Im Verständnis der Komplementarität von Massenproduktion und Massenkonsum ist daher die Kritik am ideologischen Konstrukt der postindustriellen Stadt impliziert. Die Entwicklung zum »comsumption artifact « hat das Weiterbestehen des produktiven Sektors zur Voraussetzung. Weder der Kapitalismus noch die kapitalistische Stadt können überleben, wenn sie ihre Aktivitäten von der Produktion des Werts abkoppeln, lediglich finan- 
zielle Manöver durchführen, ohne die Produktion zu erhöhen oder umzustrukturieren (Harvey, 1982: 320; 1985a: 88).

In seinem jüngsten Aufsatz (1987) erweitert Harvey seine Betrachtungen der Dialektik von Urbanisierungsprozeß und Kapitalakkumulation auf den Typus der »postmodernen Stadt«: Materielle Basis für die aktuelle Restrukturierung von Städten und Regionen ist ein neues, auf flexiblen Formen beruhendes Akkumulationsregime. ${ }^{9}$ Die Bewegung des Kapitals im Raum folgt den Bedürfnissen der industriellen Organisation: Die Postmoderne erscheint dann als das kulturelle Gewand flexibler Akkumulation. Die keynesianische Stadtstruktur zerfällt - ebenso wie das Regime der vereinheitlichten fordistischen Massenproduktion - in flexibilisierte sozialräumliche Einheiten, die den Reproduktionsbedürfnissen der neuen Ära funktional zugeordnet sind. Unterstiitzt von staatlichen Maßnahmen wie Innenstadtgentrifizierung und institutionalisierte Bündnisse von Staat und privater Wirtschaft wird die postmoderne Stadtlandschaft zunehmend von den Bedürfnissen eines Luxuskonsumismus geprägt, während die Marginalisierten den Rückzug ins Ghetto antreten (Harvey, 1987). Wo früher die Gleichmacherei des Automobils und des Einfamilienhauses gesellschaftlich akzeptiertes Prinzip einer Massenkonsumtionsökonomie war, werden jetzt Konsumtionspaläste für selektierte Märkte und eine prätentiöse Herrschaftskultur zu den Symbolen einer neuen Urbanität. In diesem Ambiente wird "symbolisches Kapital« (Bourdieu) zum Schlüsselelement. Davis hat dieses Phänomen als »Überkonsumismus « bezeichnet, womit er »die zunehmende gesellschaftliche Subvention neuer Mittelschichten durch die ständige Degradierung neugeschaffener Arbeitsplätze und die Auflösung der üblichen Standards des fordistischen Massenkonsums « meint (Davis, 1986: 94)..$^{10}$ Das Spektakel, der $\gg$ Spiele $\ll$ - Anteil in der römischen Herrschaftsformel wird zum Drehpunkt der neuen Stadt; der Kultur wächst eine noch größere Bedeutung zu, als sie für die Beherrschung des Raumes im Urbanisierungsprozeß ohnehin schon hatte (Harvey, 1987). Die »Towers of Power « in den Innenstadtbezirken dẹ Postmoderne stehen nicht länger nur als Symbole der finaziellen Macht sondern auch als spekulative Fetische einer gebrauchswertorientierten Nutzung des städtischen Raums im Wege."

Die aktuelle Restrukturierung der Städte hat historische Vorgänger. Die Rationalität der modernistisch-kapitalistischen Entwicklung kam sich mit der Zeit stets selbst ins Gehege. Neue Wellen von »kreativer Zerstörung « wurden in immer schnellerer Folge notwendig. Auch die Haussmann'sche Modernisierung von Paris schuf lediglich eine limitierte bauliche Umwelt, deren spezifische Rationalität spätere Nutzung verbat (1985b). Aspekte der postmodernen Restrukturierung der baulichen Umwelt sind ebenso historisierend wie die Fassaden ihrer Architektur: Die Deindustrialisierung von Paris im 19. Jahrhundert, die den Stadtkern der Luxusindustrie öffnete oder die Beseitigung überschüssigen Kapitals sowie überzähliger - und potentiell revoltierender - Bevölkerung aus den Städten sind Themen, die die postmoderne Urbanisierung früherer Epochen der Verstädterung entlehnt (cf. Harvey, 1985b: 119). Harvey mißtraut daher auch simplifizierenden Versuchen, die kapitalistische Entwicklung in Stufen einzuteilen. In einer Mahnung zur Vorsicht, auch gegenüber seinen eigenen Tendenzen zur Periodisierung des Urbanisierungsprozesses, stellt er heraus, daß dessen Elemente zu jedem Zeitpunkt und an jedem Ort in spezifischer Weise kombiniert werden. Eine Periodisierung nach dem Muster: Die merkantilistische Stadt moblisiert Mehrwert, die industrielle Stadt produziert, 
und die keynesianische Stadt absorbiert das Surplus, ist daher problematisch. Gerade in der aktuellen, post-keynesianischen Ära ist vorherrschend: eine Mischung aller möglichen Strategien der Kapitalakkumulation durch Urbanisierung (cf. Harvey, 1985a: 221 f.) ${ }^{12}$. Festzuhalten bleibt an diesem Punkte, daß Harvey den Urbanisierungsprozeß (und damit den gesellschaftlichen Prozeß selbst) als zeit/räumlichen begreift. Es entstehen dabei »eigenartige geographische Konfigurationen physischer und sozialer Beziehungen «, die wiederum in komplexe Formen städtischen Lebens übersetzt werden (Harvey, 1985b: 265). Der Urbanisierungsprozeß ist gleichzeitig Grundlage für die Perpetuierung des Kapitalismus und Ausdruck seiner inneren Widersprüche (Harvey, 1985b: 273).

So vielversprechend und inspirierend Harveys Versuch ist, in der oben skizzierten Weise Stadttheorie und historische Geographie zu integrieren, so wenig ist dieses Ziel bisher erreicht. Es bedarf hier detaillierter Studien gegenwärtiger Verstädterungsprozesse, also Untersuchungen städtischer Regionen, die Harveys theoretisch erarbeiteten Hypothesen unter aktuelleren Bedingungen als dem Paris des 19. Jahrhunderts, der Fallstudie in Consciousness and the Urban Experience (1985b), und in umfangreicher Form als in Harveys jüngstem Aufsatz (1987) auf den Prüfstand stellen. Dies ist um so notwendiger, als die durch Flexiblilisierung erreichte Fragmentierung des Urbanisierungsprozesses eine Mustergeschichtsschreibung der Verstädterung im Stile der Forschungen zu Manchester oder Chicago nicht zuläßt. Der Ruf nach mehr empirischer Forschung genügt freilich nicht. Vielmehr müßte die Integration verschiedener Ansätze in der Sozialwissenschaft, die die Stadtforschung befruchten können, fortgesetzt werden. Insbesondere in der Diskussion um die Entstehung der »World City « (vgl.z.B. Friedmann, 1986), in neueren Ansätzen zum Studium gegenwärtiger Restrukturierungsprozesse in städtischen Regionen (vgl. z.B. Soja, 1986; et.al. 1983) und in der Literatur, die sich mit globalen Urbanisierungstendenzen beschäftigt (vgl. z.B. Portes/Walton, 1980; Timberlake, 1985) finden sich erste Ansätze dazu. ${ }^{13}$

\section{Grundrente/Akkumulation/Klassenkampf}

Im Kern von Harveys ökonomischer Analyse der kapitalistischen Stadt findet sich seine Interpretation der Grundrente. ${ }^{14}$ Insbesondere interessiert ihn die von Marx nicht völlig geklärte Frage, warum die Grundrente im Kapitalismus weiter existiert, obwohl radikale bürgerliche Ökonomen sie als parasitär ansahen. Harvey zeigt, daß dem Bodenmarkt und damit der grundbesitzenden Klasse ${ }^{15}$ eine regulative Funktion zukommt, ohne die der Kapitalismus nicht funktionieren könnte (Harvey, 1982: 331). Damit wird die Grundrente - ohne selbst Wert zu produzieren - zum Faktor im Akkumulationsprozeß. Hergestellt ist ein komplexes soziales Verhältnis: »Das Kapital organisiert den Raum, aber der Raum hilft, das Kapital zu organisieren» (Katz, 1986: 68). Während das an den Boden gebundene Kapital als historische Voraussetzung und als Bedingung der Produktion an der Dynamisierung der Krisen im Kapitalismus beteiligt ist, werden andererseits während der Krise Investitionen in den sekundären Kapitalkreislauf zur Abschöpfung überflüssigen Kapitals aus der Sphäre der Produktion genutzt. Mit dem «spatial fix» werden dem Kapital temporär Ventile geöffnet, die allerdings im Gegenzug 
die Malaise der Überproduktionskrise nur vergrößern, weil sie die Krise auf größerer räumlicher Basis reproduzieren. Die geographische Landschaft der Kapitalakkumulation, geplant und ausgelegt, um die Umlaufzeit des Kapitals zu senken, erscheint als rationale geographische Konfiguration, die nicht lediglich Ensemble von Häusern, Straßen, etc. ist, sondern Produktionsverhältnisse verkörpert (cf. Harvey, 1985a: 32-61).

Harveys Theorie der Grundrente und der baulichen Umwelt wurde mit dem Argument kritisiert, sie entspringe zu einseitig einem »Kapitallogikansatz« (Katz, 1986). Diese Position ist repräsentativ für eine oft an Harvey geübte Kritik, die ihm mechanistisches Denken vorwirft, wenn es um die Analyse sozialer Prozesse im Raum geht: Man gewänne den Eindruck, alles würde immer so geregelt, wie das Kapital es gerade brauche. Richtig ist, daß Harvey Positionen ablehnt, die dem Klassenkampf eine von strukturellen Restriktionen relativ unbeeinträchtigte Rolle zumessen. Ihm geht es vielmehr darum zu zeigen, wie die Mechanik des Akkumulationsprozesses historisch zur Perpetuierung des Kapitalismus geführt hat, gerade indem Klassenkämpfe darin integriert werden konnten. Ökonomische Klassenkämpfe im Bereich der Produktion und der Reproduktion haben dabei bestenfalls modifizierende Wirkung: » Das Kapital mag in solchen Prozessen allgegenwärtig sein, aber es ist weder allwissend noch allmächtig « (Harvey, 1985b: 57). Forderungen der Arbeiterklasse nach mehr Kontrolle über den Arbeitsprozeß und die Reproduktionsbedingungen werden vom Kapital in der Regel mit »Lösungen« beantwortet, die seinen Fortbestand sichern:

"As labor seeks more control over the collective conditions of its existence, so capital seeks to establish collctivized forms of consumption and individual homeownership« (Harvey, 1985b: 57).

Als Beispiel für einen solchen Vereinnahmungsprozeß, der auf einem Klassenkompromiß beruht, nennt Harvey den »Genius des Fordismus «. Er erlaubte es der kapitalistischen Klasse, sich systematisch den Klassenkämpfen am Ort der Produktion zu entziehen und lieferte, durch die ihn begleitenden dramatischen Verschiebungen im Urbanisierungsprozeß, die räumliche Grundlage für eine weitere Reproduktion des Kapitals gleich mit (1985b: 254).

Harvey unterscheidet zunächst zwischen Klassenkampf (Produktion) und verlagertem Klassenkampf (Reproduktion). Später differenziert er dieses dichotomische Modell, indem er neben kollektiver Aktion im Raum und klassenbewußter politischer Aktivität individuelle politische Handlungen als niedrigste Ebene politischer Aktivität hinzufügt (1985b:60). Angestrebt ist dabei die Beseitigung der Trennungen zwischen den Bereichen und die Verlagerung ökonomischer Kämpfe in die politische Arena (1982: 449). Die Dynamik des Kapitalismus selbst hebt demnach die (seit Engels' Wohnungsfrage) als klassisch empfundene Trennung zwischen Froduktion und Reproduktion auf, indem die Schaffung neuer Konsumtionsweisen und neuer Bedürfnisse zur Überlebenslogik des Kapitals wird - auch wenn die Dichotomie zwischen beiden Sektoren als besonders scharfe erscheint (1985a: 87). Harveys Argumentation zielt daher nicht auf eine Ausbreitung von Kämpfen im ökonomischen Bereich, sei es im Betrieb oder im Stadtviertel, sondern auf eine Integration dieser Kämpfe auf der politischen Ebene, d.h. auf der Ebene der staatlichen Macht. ${ }^{16}$ 


\section{Globale Restrukturierung und städtische Politik}

In The Urbanization of Capital (1985a) versucht Harvey einem offensichtlichen Defizit seiner Theorie zu begegnen - der Vermittlung von ökonomischen Prozessen durch die politische Sphäre in der Stadt. Als Ausgangspunkt dienen ihm dabei die vom aktuellen ökonomischen Restrukturierungsproze $B$ geschaffenen neuen Bedingungen. In einer restrukturierten globalen Ökonomie werden städtischen Regionen neue Bedeutungen innerhalb der städtischen Hierarchie zugemessen (cf. Logan/Molotch, 1987: 257 ff.). Während einerseits dieser Einordnungsprozeß selbst aktives Handeln von städtischen Wachstumskoalitionen voraussetzt, welche die abstrakte Dynamik des globalen Kapitals regional verträglich zu machen versuchen, schaffen andererseits die neuen sozialräumlichen Konfigurationen einen veränderten Schauplatz für städtische Politik. - Harvey benennt vier Bereiche, in denen Städte im gegenwärtigen Restrukturierungsprozeß um ihre Position in der innerstädtischen Hierarchie kämpfen müssen: internationale Arbeitsteilung, globaler Wettbewerb in der räumlichen Teilung der Konsumtion, Konkurrenz um Kommandofunktionen in der Weltökonomie und um redistributive Zahlungen aus der Staatskasse.

Das individuelle Wettbewerbsergebnis wird dabei ganz entscheidend davon abhängen, welche spezifische Struktur der lokale Arbeitsmarkt aufweist (oder welchen Veränderungen er sich zu unterwerfen bereit ist). Dabei kann es relevant sein, wie flexibel die am lokalen Markt orientierten Sektoren sind, wie stark neue Technologien in vorhandenen Branchen Eingang finden, welche innerstädtischen Standortvorteile für einzelne Bereiche der Ökonomie vorhanden sind etc. (Harvey, 1985: 138). Die interne Diversifizierung, die Polarisierung und Segmentierung der städtischen Arbeits- und Konsumtionsmärkte, der Stadtstruktur und letztlich der politischen Sphäre werden zur Existenzvoraussetzung für das Bestehen im internationalen Wettbewerb. Territoriale Arbeitsteilung ist schließlich kein regionales Problem mehr, sondern die Konkretisierung der abstrakten Bedürfnisse des internationalisierten Kapitals auf Stadt-Teil-Ebene. Auf dieser Ebene erscheint die Genesis eines neuen Akkumulationsregimes als interne Restrukturierung urbaner Regionen. Widersprüche entstehen hier zunächst zwischen lokalen Kapitalinteressen und denen des internationalen Kapitals. Diese Widersprüche werden zum Verhandlungsgegenstand städtischer Politik, insbesondere auf dem Gebiet der Wirtschaftsentwicklung. Die allgemeine Stabilität des Kapitalismus ist von seiner lokalen Integrationsfähigkeit abhängig (Harvey, 1982: 442 f,; 1985a: 223). Städtische Politik ist daher mit der Herstellung einer strukturierten Kohärenz befaßt, also mit einem Ensemble von Arbeitsmärkten und Reproduktionszusammenhängen auf der Basis einer lokal spezifischen Kombination von Technologien. - Ein ähnliches Modell hat Mike Davis kürzlich in einer historischen Studie von Los Angeles vorgeschlagen. Darin plädiert er für eine Anwendung des Konzepts des »Akkumulationsregimes « auf subnationaler Ebene, um »raum-spezifische Subsumtionsweisen der Arbeit und der Organisation von Klassenkämpfen identifizieren zu können « (Davis, ohne Datum: 60). Die spezifische ökonomische Basis einer Stadt, ihre soziale Formation und ihr »System räumlicher Organisation, das Produktion, Konsumtion und politische Herrschaft koordiniert, « können somit im speziellen Fall zum Gegenstand der Untersuchung gemacht werden und den ortsspezifischen Handlungsspielraum politischer Akteure definieren helfen (ibid.: 61). 
Bei Anwendung solcher Modelle besteht allerdings die Gefahr, die Konkurrenz zwischen Stadtregionen als den wesentlichen räumlichen Widerspruch zu sehen. Doch die Tendenz zur Herstellung einer strukturierten Kohärenz führt nicht zu einer monolithischen Funktionalität von städtischen Räumen innerhalb der internationalen Arbeitsteilung, sondern zu neuen innerstädtischen Widersprüchen: Teile eines einzelnen spezifischen städtischen Arbeitsmarktes können hochkarätige Kontroll- und Managementaufgaben innerhalb der globalen Ökonomie wahrnehmen, während andere Sektoren am gleichen Ort in den Strudel einer Peripherisierung der Arbeit gezogen werden (Sassen-Koob, 1984). Die Subsumtion immer größerer Teile der Arbeiterklasse in den Metropolen unter Produktionsbedingungen, die man dort als ausgestorben betrachtet hatte (sweatshops, Tagelöhner), geht einher mit einer enormen Steigerung der Arbeitsproduktivität - durch den massiven Einsatz neuer Technologien in allen Produktionsbereichen (cf. Castells, 1985). Der Restrukturierungsprozeß erscheint hier also als räumlich definierter Mechanismus zur Steigerung des absoluten und relativen Mehrwerts. Im gleichen Maße werden die fordistischen Reproduktionsstrukturen zugunsten einer Diversifizierung des gesellschaftlichen Konsums abgebaut. Diese Verschiebung ist gekennzeichnet durch einen Rückzug hinter das »sozialdemokratische Minimum« in der gesellschaftlichen Konsumtion (Katznelson, zitiert in: Ross/Trachte, 1983: 407) und durch die Herausbildung neuer Mittelklassen für den Luxuskonsum (Davis, 1986). So gesehen liefern die Konzepte von Harvey und Davis eine Hilfe zum Verständnis der sozio-ökonomischen Restrukturierung der Städte als (lokalen) politischen Prozeß. ${ }^{16}$

\section{Klasse, Territorium und lokale Politik}

Aufgabe lokaler Politik ist nicht nur die Integration einer Stadtregion in die globale Ökonomie sondern ebenso die Vermittlung und Beherrschung innerstädtischer Widersprüche. In früheren Schriften operierte Harvey mit einem simplen, dichotomischen Begriff von Klasse und »community (Gemeinde im Stadtteil). In Consciousness and the Urban Experience differenziert er diese Begriffe und setzt sie in ein neues Verhältnis. »Community«, so meint er, habe eine klassenspezifische Bedeutung (1985b: 162). Die »Gemeinde « der nationalen und internationalen Bourgeoisie verfügt naturgemäß über größere territoriale Macht als die oft bornierten und limitierten Gemeinden der Arbeiterklasse (1985b: 166). Revolutionen, Krisen und sozio-ökonomische Restrukturierung setzen die Grenzen der Begriffe Klasse und »community « in territorialer und sozialer Hinsicht in Bewegung. Im Prozeß der sozialräumlichen Restrukturierung entstehen neue soziale Einheiten, die mit neuen räumlichen Konfigurationen korrelieren. »Community « als typisch urbane gesellschaftliche Form ist daher kein unhistorisches Organisationsprinzip, sondern wird durch Klassenverhältnisse definiert und modifiziert. Ihr Charakter ist widersprüchlich: Einerseits ist zur Aufrechterhaltung kapitalistischer Klassenverhältnisse deren Mystifizierung mit Hilfe einer »operativen geographischen Konzeption von 'community" notwendig " (Harvey, 1985a: 148). Andererseits kann die städtische Gemeinde auch von der Arbeiterklasse als »offensive Waffe im Klassenkampf « eingesetzt werden (Harvey, 1978). Entscheidend für Harveys Verständnis städtischer Raum/Klassenstruktur ist dabei die Vorstel- 
lung, daß unterschiedliche »communities« über ganz verschiedene Ressourcen verfügen, um Raum überwinden und beherrschen zu können. Thre Mobilität ist Faktor ihrer jeweiligen Klassenbindung und der andauernden Klassenkämpfe im Raum (Harvey, 1987; Logan/Molotch, 1987). Die Dependenz bestimmter Orte von übergeordneten Strukturen scheint mit der Mobilität des Kapitals zu wachsen, denn das räumlich-soziale Netz des Kapitalismus wird durch Dispersion und Flexibilisierung fester gefügt. Harvey erkennt zudem, daß Prozesse der Vergesellschaftung und Bewußtseinsbildung in der urbanen Gesellschaft komplexer sind als daß sie sich in einem dichotomischen Modell ausdrücken ließen. Klasse und städtische Gemeinde sind letztlich - neben Familie und Staat z.B. - nur zwei von mehreren primären Orten der Bewußtseinsformation (1985b: 252). Die Urbanisierung des Bewußtseins, also der Prozeß der Bewußtseinsformation durch Urbanisierung, ist ständigen Restrukturierungsschüben ausgesetzt (1985b:252). Die spezifische Art und Weise, in der Individuen in diesem Prozeß verschiedene Konzeptionen internalisieren, tragen zur Konfusion in der ideologischen und politischen Sphäre bei (1985b: 262). Dies verhindert in den meisten Fällen städtischer sozialer und politischer Auseinandersetzungen klare Identitäten der Akteure.

Mit einem solchen soziologischen Modell der Struktur und des politischen Milieus der Stadt will Harvey den »Ouvrierismus « überwinden, der einigen seiner früheren Schriften eigen war - wo er die städtische Bevölkerung auf eine undifferenzierte Arbeiterklasse zu reduzieren tendierte. ${ }^{17}$ Allerdings bleibt noch einiges zu tun übrig, um die aktuellen städtischen Restruktierungsprozesse in ein umfassenderes Modell zu integrieren und den politischen Charakter der Restrukturierung zu verstehen. Es genügt nicht zu zeigen, daß die Kommodifizierung von traditionellen Strukturen gegenseitiger Hilfe in und den Wertabfluß aus vielen verarmenden Stadtteilen mit dem neuen Regime der flexiblen Akkumulation konsistent ist. Vielmehr müssen gerade die Funktionen einzelner sozialräumlicher Fragmente im städtischen Gesamtzusammenhang in ihrem Bezug auf die globale Ökonomie bestimmt werden. Der Prozeß der Informalisierung ist zudem äußerst differenziert und die Kreation neuer Vergesellschaftungsformen ambivalent - wie die Untersuchung von »mediating structures« und »public-privatepartnerships» in den USA beweist (cf. Mayer, 1986). Wegen der äußerst schwierig zu sezierenden und kaum zu verallgemeinernden lokalen Ausformung der Dynamik globaler ökonomischer Restrukturierungsprozesse ist die Formulierung von politischen Programmen gegenüber der Stadt eine härtere Nuß, als es bei Harvey (1987) erscheinen mag.

Harveys politische Formulierungen sind in Abgrenzung zu einer Reihe von maßgeblichen Theoretikem auf dem Gebiet der Urbanistik entstanden. Fast verwundert stellt er fest, daß viele Autoren auf der Linken gerade zum gegenwärtigen Zeitpunkt der marxistischen Theorie den Rücken kehren - wo die Krise des Kapitalismus sich doch mit gigantischen Umwälzungen der Produktivkräfte und der gesellschaftlichen Beziehungen bemerkbar macht (1985b: 267). Explizit ist darum seine Opposition zu Theoretikern wie Saunders (1981), Mollenkopf (1983) oder Castells (1983), die - aus unterschiedlichen Gründen - davon überzeugt sind, daß es nutzlos sei, eine rein materialistische, in der marxistischen Tradition stehende Interpretation städtischer Konflikte zu versuchen (1985a: 125). Deutlicher noch als die genannten Autoren grenzen sich seit neuestem Autoren aus dem Bereich der »New Urban Ecology «(Feagin, 1986) vom Marxismus in der Stadttheorie ab. Gottdiener (1985) postuliert beispielsweise, daß die Idiosyn- 
krasie der aktuellen Urbanisierungstendenzen (vor allem der polynuklearen Strukturen in den USA) von der marxistischen Theorie nicht erfaßt werden können. Harveys Verdienst ist es sicherlich, die gegenwärtige Restrukturierung der politischen Form des fordistisch-keynesianischen Vergesellschaftungsmodus (cf. Hirsch/Roth, 1986) mit einer elaborierten und umfassenden Theorie der Urbanisierung, deren Kategorien er aus dem Marxismus entwickelt hat, zu verbinden. Allerdings scheint bei vielen seiner Kritiker, auch bei solchen, die seinen Ansatz akzeptieren (z.B. Logan/Molotch, 1987), das Mißverständnis vorzuherrschen, bei Harveys »Orthodoxie« handele es sich nicht um eine politische Entscheidung für einen gewissen Standpunkt, sondern um einen Mangel, den es zu beseitigen gelte. Folglich besteht eine Spannung zwischen Harveys Standpunkt und denjenigen Positionen auf der Linken, die den historischen und theoretischen Ablösungsprozeß von der marxistischen Orthodoxie und die Öffnung gegenüber neuen gesellschaftlichen Konfliktfeldern und Deutungsmustern begrüßen (cf. Hirsch/ Roth, 1986: 10-45; 168-240).

Harvey ordnete dem lokalen politischen Sektor eine spezifische Rolle im aktuellen Restrukturierungsprozeß zu. Er geht ähnlich wie Lefebvre (1972) davon aus, daß »soziale, ökonomische und politische Prozesse auf der städtischen Analyseebene eine besondere Bedeutung haben« (1985a: 163). Dort liegt die Schnittstelle von täglichem Handeln und globalen Prozessen (vgl. Rodenstein 1986). Es ist dies zugleich auch eine Kritik an raumlosen Konzeptionen in der marxistischen Tradition. Zudem ist in Harveys Augen die Formation sozial-räumlicher Konfigurationen in städtischen Regionen instrumental für das Verständnis der Formation des Staates. Räumliche Beziehungen und geographische Entwicklungen innerhalb der Evolution einer Akkumulationsperiode erklären seiner Meinung nach die Entstehung bestimmter Staatsfunktionen (lokal, regional, national, supra-national; 1985c: 143 f.). Globale Klassenauseinandersetzungen lösen sich in die verschiedensten interterritorialen Konflikte auf. Die Notwendigkeit zur Bildung und Auflösung von Staatsapparaten im Kapitalismus ist daher im Kontext der Bildung und Auflösung geographischer Klassenbündnisse zu sehen (1985c: 152). Der Staat, der in diesem Prozeß der Formation einer strukturierten Kohärenz mit spezifischen eigenen Interessen ausgestattet ist, wird zum »Schlüssel für den Ausdruck der Tendenz, regionale Klassenbündnisse zu schaffen « (1985c: 152). Der Vorstellung, daß städtische Politik mit der Regulation und Integration der strukturierten Kohärenz befaßt ist, ist die Kritik solcher lokalen Politikmodelle implizit, die den Handlungsspielraum städtischer Politik auf den Bereich der kollektiven Konsumtion beschränkt sehen (cf. Harvey, 1985a: 125 ff.). Darïber hinaus ist damit gesagt, daß--obwohl die Funktionen des lokalen Staates an die Bedürfnisse des kapitalistischen Systems gebunden sind - die Zuständigkeiten lokaler Politik nicht einseitig von der globalen Ebene definiert werden. Sie sind selbst Gegenstand lokaler politischer Klassenkämpfe: Worum es in der kommunalen Politik überhaupt gehen soll, ist ebenso umkämpft wie die eigentliche lokale Politik selbst (cf. Fincher, 1981). Der lokale Raum hat folglich eine gewisse Autonomie. Er ist ein Treibhaus innerkapitalistischer Innovation, ${ }^{18}$ an der die gesamte städtische Gesellschaft - auch die städtischen sozialen Bewegungen - beteiligt ist (sind). Diese Situation ist explosiv:

»The urban region either submits to the forces that created it or becomes the hearth of a revolutionary movement« (1985a: 162). 


\section{Das unfertige Projekt einer materialistischen Stadttheorie}

Harvey genügt seinem eigenen Anspruch, die »Oberflächenphänomene« städtischer Restrukturierung in allen Bereichen des urbanen Lebens (Wohnungsfrage, Gesundheitsfürsorge, soziale Dienstleistungen, Erziehung, Umwelt, etc.) an die ihnen »unterliegenden und oft obskuren Bedürfnisse der Kapitalakkumulation und der Perpetuierung der herrschenden Klassenverhältnisse in der Produktion « zu knüpfen. Er erkennt die Spannungen, die durch die städtische Restrukturierung zugunsten flexibler Formen der Akkumulation im urbanen Kontext entstehen. Obwohl er beteuert, daß die gegenwärtigen Verschiebungen innerhalb des kapitalistischen Systems ebenso prä-sozialistisch wie post-modern sind, ist er der Auffassung, daß sich das kapitalistische System insgesamt nicht in der Krise befindet. Die Handlungsspielräume progressiver städtischer Politik sind entsprechend limitiert (Harvey, 1987). Der städtische politische Raum bleibt für Harvey in erster Linie die Hochburg derjenigen Kräfte, die die Perpetuierung der kapitalistischen Verhältnisse verfolgen. Dies gilt um so mehr, als der Kapitalismus durch die Produktion von Raum am Leben erhalten wird (1985b: 265). Allerdings macht die Zersplitterung des politischen Bewußtseins, welche durch die Segregation des städtischen Raums nach den Grenzen der flexiblen Akkumulation noch verstärkt wird, die Verschiebung vom »fragmentierten Pluralismus zu einer mehr klassenorientierten Politik « in der amerikanischen Stadt zu einem schwierigen Projekt (Harvey, 1985: 225).

Sicherlich gibt es eine Reihe von Defiziten in Harveys Ansatz, vor allem im Bereich der Vermittlung zwischen ökonomischen Prozessen und politischer Formkrise, im Bereich der Schnittstelle zwischen institutionalisierter Staatsmacht und sozialen Bewegungen oder in bezug auf das Verhältnis zwischen dem lokalen Staat und sublokalen Strukturen. Als umfassender Ansatz eines weitergehenden Projekts materialistischer Stadtheorie ist David Harveys Werk jedoch das Überzeugendste, was die Disziplin derzeit zu bieten hat.

\section{Anmerkungen}

1 Zur kontroversen Problematik von Gegenstand und Aufgabe einer spezifisch stadtorientierten Sozialwissenschaft cf. Castells, 1977; Pickvance, 1976; Kraemer/Neef, 1985; Lefebvre, 1972; Harvey, 1973; Zukin, 1980, Saunders, 1981.

2 Als Grundlage einer materialistischen Stadtforschung gelten im allgemeinen Engels' Texte Zur Lage der arbeitenden Klassen in England und Zur Wohnungsfrage. Marx selbst hatte der Frage des Raumes aus unterschiedlichen Gründen wenig Bedeutung zugemessen. Ein Hauptgrund war die Relevanz der Zeit als Medium der Messung der Arbeitszeit, also der Grundlage der Mechanik der Ausbeutung selbst, für die bürgerliche Ökonomie. Auch mag eine bewußte Zurïckweisung der Hegel'schen Position maßgebend gewesen sein. Hegel hatte Kolonialismus und Imperialismus als externe Lösungen für interne Widersprüche der kapitalistischen Länder für möglich gehalten (Harvey, 1985a: 52; zur Rolle des »spatial fix « bei Harvey siehe unten). Auch war Marx die Glorifizierung des territorialen Nationalstaates durch Hegel suspekt, weswegen er den Primat der revolutionären Zeit äber den Raum favorisierte, um den Klassenkonflikt zu entmystifizieren (cf. Soja, 1985: 105-107). Harvey selbst versucht, die zeitliche Dimension im Denken von Marx mit der Imperialismustheorie Lenins zu verbinden (Harvey, 1985c). Zum Problem der Stadt »im marxistischen Denken« cf. Lefebvre, 1975. 
3 Der Begriff »urban process « bereitet ebenso Schwierigkeiten in der Übersetzung wie »built environment« (bauliche Umwelt), »spatial fix« und »community« (städtische Gemeinde). Die hier vorgeschlagenen Übersetzungen sind nur Annäherungen an die englischen Termini.

4 Für eine deutlich unterschiedliche marxistische Postiion cf. Lefebvre, 1972. Siehe auch Castells' Revision seiner ursprünglichen Position: 1983, 1984.

5 Für einen Überblick aktueller Positionen cf. Gregory/Urry, 1985. Für die westdeutsche Diskussion cf. Prigge, 1986.

6 Der Begriff der Krise spielt eine zentrale Rolle in Harveys Verständnis des kapitalistischen Akkumulations/Urbanisierungsprozesses. In The Limits to Capital (1982) erstellt er eine Krisentypologie. Vgl. auch 1985a: 11-13.

7 Finanzkapital wird hier verstanden als Einheit von Banken-, Handels- und Industriekapital, wobei davon auszugehen ist, daß es sich dabei um eine spannungsreiche und widersprüchliche Verbindung handelt. Das gleiche gilt für die Klasse, die das verbindende strategische Zentrum von Finanz und Produktion darstellt. Auch hier haben wir es mit einer sehr instabilen Konfiguration zu tun. Die Rolle des Staates als »Herz des strategischen Kontrollzentrums für die Zirkulation des zinstragenden Kapitals « ist herausragend (Harvey, 1982: 319 ff.). Das Vorhandensein eines funktionierenden Kapitalmarkts und des Staats als Financier sind die unmittelbaren Voraussetzungen des Kapitalflusses in den sekundären Kreislauf (Harvey, 1985 a: 7).

8 Zur Beschreibung der Konsequenzen dieser Dynamik für den Vergesellschaftungs- und Bewußtseinsbildungsprozeß siehe Harvey 1985b: 254 ff.

9 Harveys Rekurs auf Konzepte der Marxschen politischen Ökonomie stattet ihn mit einer traditionell marxistischen Terminologie und mit einer Reihe von Kategorien aus, die im Verbund mit seinen eigenen Konzepten als der paradigmatische Werkzeugkoffer der politökonomisch orientierten Stadttheorie gelten können. Konzepte aus anderen Bereichen der sozial- oder wirtschaftswissenschaftlichen Diskussion wie »flexible Akkumulation« und »Akkumulationsregime« verwendet Harvey meist »in Anlehnung « an, selten deckungsgleich mit den Urhebern diese Begriffe.

10 Harvey und Davis bezichen sich beide auf neueste amerikanische Entwicklungen. Vgl. auch Jameson, 1984; Davis, 1985.

11 Zum Konzept von Tauschwert und Gebrauchswert im städtischen Kontext cf. Logan/Molotch, 1987.

12 Die Betonung der komplexen Struktur von Urbanisierungsstrategien ist deshalb von Bedeutung, wei1 marxistische Theoretiker oft der simplizifierenden Periodisierung bezichtigt werden. Vgl. Gottdieners (1985: 76) überzogene Kritik an Gordon. Zum Problem der Periodisierung vgl. Jessop, 1983.

$13 \mathrm{Vgl}$. für die westdeutsche Situation cgf. Friedrichs/Häussermann/Siebel, 1986; Blanke/Evers/Wollmann, 1986.

14 Für einen Überblick über die theoretische Debatte cf. Fine. 1979; Edel, 1976; Gottdiener, 1985; Harvey, 1982: $330 \mathrm{ff}$; Brede/Dietrich/Kohaupt, 1976.

15 Kritiker Harveys haben auf das Fehlen einer solchen Klasse in den USA hingewiesen (Gottdiener, 1985: $160 \mathrm{ff}$.).

16 Zum Konzept der Restrukturierung cf. Soja, 1984; Smith/Feagin, 1987.

17 Eine Tendenz, die ich im Gegensatz zu Kritikern Harveys wie Gottdiener (1987) im Falle der herrschenden Klassen nicht entdecken kann.

18 Harvey unterscheidet gegenwärtig vier Dimensionen dieses Prozesses: 1) die traditionelle Wachstumsmaschinerie; 2) die Stadt als Innovationszentrum; 3) die Stadt als kollektive Einheit im internationalen Wettbewerb; 4) die Stadt als aktive Komponente in der Restrukturierung geopolitischer Zusammenhänge (1985a). 


\section{Literatur}

Aglietta, Michel (1979): A Theory of Capitalist Accumulation. London: Verso

Badcock, Blair (1984): Unfairly Structured Cities. Oxford: Basil Blackwell

Blanke, B./A. Evers/H. Wollmann (Hg.) (1986): Die Zweite Stadt, Leviathan-Sonderheft 7

Brede, Helmut/Barbara Dietrich/Bernhard Kohaupt (1976): Politische Ökonomie des Bodens und Wohnungsfrage. Frankfurt: Suhrkamp

Castells, Manuel (1985): »High Technology, Economic Restructuring, and the Urban-Regional Process in the United States «, in: M. Castells (Hg.), High Technology, Space and Society. Beverly Hills: Sage

Ders. (1984): »Space and Society: Managing the New Historical Relationships«, in: M.P. Smith (Hg.), Cities in Transformation. Beverly Hills: Sage

Ders. (1983): The City and the Grassroots. Berkeley and Los Angeles: University of California Press

Ders. (1977): Die kapitalistische Stadt. Berlin

Davis, Mike (1986): Phoenix im Sturzflug. Berlin: Rotbuch

Ders. (1985): »Urban Renaissance and the Spirit of Postmodernism«, in: New Left Review Nr. 151 (Mai/ Juni): 106-113

Ders. (ohne Datum): »Sunshine and the Open Shop: The Urbanization of Los Angeles - 1880-1930«, unveröffentl. Manuskript (vgl. ders. (1987): Chinatown, Part Two? The 'Internationalization' of Downtown Los Angeles, in: New Left Review, No. 164 (1987), S. 65-86

Dunleavy, P. (1980): Urban Political Analysis. London: Macmillan

Edel, Matthew (1977): »Rent Theory and Labor Strategy«, Review of Radical Political Economics 9: 115

Feagin, Joe R. (1986): »Toward a new Urban Ecology«, in: Contemporary Sociology 15,4: 531-533

Fine, Ben (1979): »On Marx's theory of Agricultural Rent«, in: Economy and Society 8: 243-250

Fincher, Ruth (1981): »Analysis of the Local Level Capitalist State «, in: Antipode 13,2: 25-31

Friedmann, John (1986): »The World City Hypothesis《, in: Development and Change 17: 69-83

Friedrichs, J./Hartmut Häussermann/Walter Siebel (Hg.) (1986): Süd-Nord-Gefälle in der BRD? - Sozialwissenschaftliche Analyse. Opladen: Westdeutscher Verlag

Gottdiener, Mark (1987): »Urbanization, Consciousness and the limits of capital logic«, in: International Journal of Urban and Regional Research 11,1: 125-131

Ders. (1985): The Social Production of Urban Space. Austin: University of Texas Press

Gregory, Derek/John Urry (Hg.) (1985): Social Relations and Spatial Stuctures. London: Macmillan

Harvey, David (1987): »Flexible Accumulation through Urbanization«, in: Prokla 69

Ders. (1985): The Urbanization of Capital. Baltimore, Md.: Johns Hopkins University Press

Ders. (1985b): Consciousness and the Urban Experience. Baltimore, Md.: Johns Hopkins University Press

Ders. (1985c): »The Geopolitics of Capitalism«, in: Gregory/Urry: 128-163

Ders. (1982): The Limits of Capital. Oxford: Basil Blackwell

Ders. (1981): »The Spatial Fix - Hegel, von Thuenen, and Marx«, in: Antipode 13,2: 1-12

Ders. (1978): »The Urban Process Under Capitalism«, in: International Journal of Urban and Regional Research 2,1: 101-131

Ders. (1977): »Labor, Capital and Class Struggle Around the Built Environment in Advanced Capitalist Societies«, Politics and Society 6: 265-295

Ders. (1975a): »The Geography of Capitalist Accumulation: A Reconstruction of the Marxian Theory«, in: Antipode 7,2: 9-21

Ders. (1975b): »The Political Economy of Urbanization in Advanced Capitalist Societies: The Case of the United States«, in: G. Gappert/H.M. Rose (Hg.) The Social Economy of Cities. Beverly Hills, Sage: $119-163$

Ders. (1973): Social Justice and the City. London: Edward Arnold

Ders. (1969): Explanation in Geography. London: Edward Arnold

Ders./L. Chatterjee (1974): »Absolute Rent and the Structuring of Space by Financial and Governmental Institutions«, in: Antipode 6: 22-36 
Hirsch, Joachim/Roland Roth (1986): Das neue Gesicht des Kapitalismus: Vom Fordismus zum PostFordismus. Hamburg: VSA

Jameson, Frederic (1984): »The Cultural Logic of Late Capital«, in: New Left Review Nr. 146 (Juli/August): 53-92

Jessop, Bob (1983): »Accumulation Strategies, State Forms, and Hegemoncic Projects«, in: Kapitalistate Nr. 10/11:89-111

Katz, Steven (1986): »Towards A Sociological Definition of Rent: Notes on David Harvey's The Limits to Capital«, in: Antipode 18,1: 64-78

Kraemer, J./R. Neef (Hg.) (1985): Krise und Konflikt in der Großstadt im entwickelten Kapitalismus. Basel: Birkhäuser

Lefebvre, Henri (1976): The Survival of Capitalism. London: Alison and Busby

Ders. (1975): Die Stadt im marxistischen Denken. Ravensburg

Ders. (1972): Die Revolution der Städte. München

Logan, John R./Harvey L. Molotch (1987): Urban Fortunes: The Political Economy of Place. Berkely und Los Angeles: University of California Press

Mayer, Margit (1986): Soziale Bewegungen in der Stadt: Fine vergleichende Untersuchung von Veränderungsprozessen im Verhältnis zwischen städtischen Bewegungen und Staat in den Vereinigten Staaten von Amerika und der BRD. Habilitationsschrift, Frankfurt am Main

Dies./Roland Roth/Volkhard Brandes (Hg.) (1978): Stadtkrise und soziale Bewegungen: Texte zur internationalen Entwicklung. Köln/Frankfurt: Europäische Verlagsanstalt

Mollenkopf, John (1983): The Contested City. Princeton: Princeton University Press, 1983

Pickvance, Chris G. (1976): Urban Sociology. London

Portes, Alejandro/John Walton (1981): Labor, Class and the International System. New York: Academic Press

Prigge, Walter (1986): Zeit, Raum und Architektur: Zur Geschichte der Räume. Köln: Deutscher Gemeindeverlag

Rodenstein, Marianne (1986): »Zum Verhältnis von Stadt und Staat in der derzeitigen Krise «, Vortrag gehalten am FB Gesellschaftswissenschaften an der JWG Universität Frankfurt am Main

Ross, Robert/Kent Trachte (1983): »Global Cities, Global Classes: The Peripheralization of Labor in New York City«, in: Review 6,3: 393-431

Sassen-Koob, Saskia (1984): »The New Labour Demand in Global Cities«, in: Michael Peter Smith (Hg.), Cities in Transformation. Beverly Hills: Sage: 139-171

Saunders, Peter (1981): Social Theory and the Urban Question. New York: Holmes and Meier

Scott, Allen J./Michael Storper (Hg.) (1986): Production, Work, Territory: The Geographical Anatomy of Industrial Capitalism. Boston: Allen und Unwin

Smith, Michael P./Joe Feagin (Hg.) (1987): The Capitalist City: Global Restructuring and Community Politics

Soja; Edwar W. (1985): »The spatiality of Social Life: Towards a Transformative Retheorization«, in: Gregory/Urry: $90-127$

Ders. (1984): »L.A.'s the Place: Economic Restructuring and the Internationalization of the Los Angeles Region «, Vortrag gehalten beim Annual Meeting of the American Sociological Association, 27. 31. August 1984, San Antonio

Ders./Rebecca Morales/Götz Wolff (1983): »Urban Restructuring: An Analysis of Social and Spatial Change in Los Angeles«, in: Economic Geography 59,2: 195-230

Storper, Michael/Richard Walker (1983): »The Theory of Labor and the Theory of Location«, in: International Journal of Urban and Regional Research 7,1: 1-43

Timberlake, Michael (Hg.) (1985): Urbanization in the World Economy. New York: Academic Press Zukin, Sharon (1980): »The Cutting Edge: a Decade of the New Urban Sociology«, in: Theory and Society 9 